\title{
Singular magnetic dilution behavior in a quasicrystal approximant
}

\author{
Takayuki Shiino $\odot,{ }^{1, *}$ Fernand Denoel, ${ }^{1, \dagger}$ Girma Hailu Gebresenbut $\odot,{ }^{2}$ Deep Chandra Joshi $\odot,{ }^{1}$ Yu-Chin Huang, ${ }^{2}$ \\ Cesar Pay Gómez, ${ }^{2}$ Ulrich Häussermann, ${ }^{3}$ Andreas Rydh $\odot,{ }^{4}$ and Roland Mathieu $\oplus^{1}$ \\ ${ }^{1}$ Department of Materials Science and Engineering, Uppsala University, Box 35, 75103 Uppsala, Sweden \\ ${ }^{2}$ Department of Chemistry, Ångström Laboratory, Uppsala University, 75121 Uppsala, Sweden \\ ${ }^{3}$ Department of Materials and Environmental Chemistry, Stockholm University, 10691 Stockholm, Sweden \\ ${ }^{4}$ Department of Physics, Stockholm University, 10691 Stockholm, Sweden
}

(Received 20 June 2021; revised 1 October 2021; accepted 19 November 2021; published 8 December 2021)

\begin{abstract}
We report the effect of magnetic dilution on the physical properties of $\left(\operatorname{Gd}_{1-x} \mathrm{Y}_{x}\right) \operatorname{Cd}_{6}$ approximant crystals (ACs), close siblings of their corresponding quasicrystal (QC). Compared to the pure system $\mathrm{GdCd}_{6}$, we observe remarkable changes in the thermodynamic and magnetic bulk properties near and below the static-ordering temperatures from diluting the magnetic $\mathrm{Gd}$ atoms with nonmagnetic $\mathrm{Y}$ atoms by only $1-3 \%(x=0.01-0.03)$. On the other hand, the corresponding QC system exhibits a monotonic change in its spin-glass behavior upon the magnetic dilution. We discuss the origin of the magnetic-dilution behavior in the present AC system in terms of possible magnetic frustration and short-range magnetic correlation that can be linked to its peculiar structure.
\end{abstract}

DOI: 10.1103/PhysRevB.104.224411

\section{INTRODUCTION}

In condensed matter physics, materials' physical properties can be dramatically changed by a small presence of defects or impurities, as exemplified by the effect of chemical doping in semiconductors and strongly correlated electron systems. In the field of magnetism, a very informative question relates to how the cooperative/collective behavior of interacting magnetic moments is influenced by defects or impurities.

Quasicrystals (QCs) are unique crystals that have longrange aperiodic order with diffraction symmetries forbidden in conventional crystals (e.g., fivefold rotational symmetry). Icosahedral QCs have attracted a great deal of attention due not only to the elegance of their quasiperiodicity and crystal structures [1] but also to their unique physical phenomena such as unusual quantum criticality [2] and an intriguing spin excitation spectrum of neutron scattering [3]. Thus their magnetic properties are expected to be very peculiar compared to conventional periodic crystals.

Approximant crystals (ACs) are periodic and compositionally similar crystals related to their corresponding QCs [4]. The most common type of QCs and ACs are Tsai-type [5] ones, whose building units are clusters made of four concentric polyhedral shells followed by a tetrahedral unit at the cluster center [see Fig. 1(a)]. The clusters are arranged periodically in ACs and aperiodically in QCs. To understand the

\footnotetext{
*takayuki.shiino@angstrom.uu.se

†fernand.denoel@angstrom.uu.se
}

Published by the American Physical Society under the terms of the Creative Commons Attribution 4.0 International license. Further distribution of this work must maintain attribution to the author(s) and the published article's title, journal citation, and DOI. Funded by Bibsam. structures and physical properties of QCs, ACs have played an important role by providing the local structural information [1,6-8]. Tsai-type $1 / 1 \mathrm{AC}$, which is the most abundant and commonly studied, is of special interest due to its periodicity and unique polyhedral structures (see Ref. [9] for the meaning of ' $1 / 1$ '). Interestingly, it includes all the Platonic solids (with certain distortion) in its crystal structure. From a magnetic point of view, Tsai-type 1/1 ACs are described as follows. Their magnetism is based on rare-earth (RE) magnetic moments residing on the icosahedral shell, which are interacting through the Ruderman-Kittel-Kasuya-Yosida (RKKY) type indirect exchange interaction. By looking only at the magnetic RE sites, the system can be viewed in two equivalent ways: either by a periodic arrangement of magnetic icosahedral units [10] [see Fig. 1(b)] or by distorted corner-sharing octahedral units [11] [see Fig. 1(c)], both of which potentially yield geometrical magnetic frustration. The magnetic behaviors of Platonic solids of higher-order symmetry (i.e., octahedron and icosahedron) are not commonly investigated. As of now, spinglass (SG) -like behavior [10,12-14] and static long-range ordering [12,15-19] have been observed in most magnetic ACs, but intriguing noncoplanar magnetic-order structures [20,21], heavy-fermion behavior [13,22], and pressure-driven quantum critical behavior [23] have also been reported. However, the possible geometrical frustration in such a unique magnetic geometrical network without chemical disorder remains to be clarified.

In this paper, we focus on the $\mathrm{GdCd}_{6} 1 / 1 \mathrm{AC}$. The system has a good localization of $4 f$ electrons and large magnetic moments with antiferromagnetic interactions [24]. Unlike the ternary AC compounds most commonly studied, the present system does not have intrinsic site-disorder randomness; see the Supplemental Material [25], which includes Ref. [26]. According to a previous report by Mori et al. [24], $\mathrm{GdCd}_{6}$ exhibits a peculiar magnetic ordering behavior. 


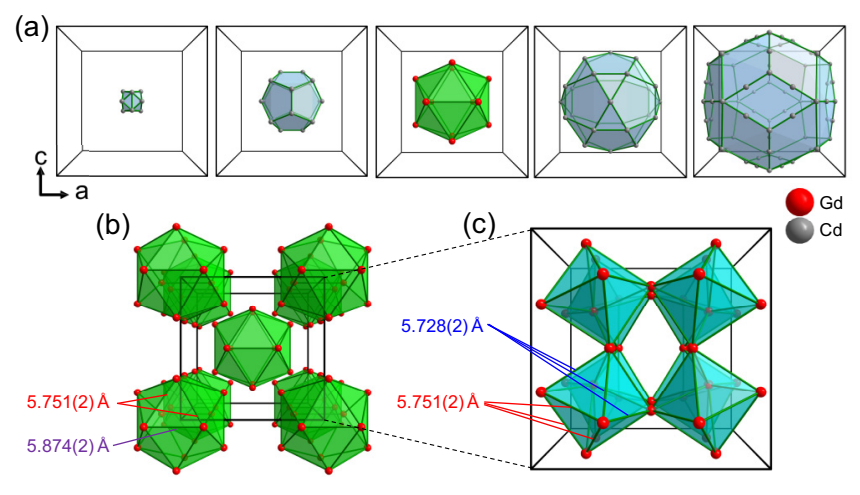

FIG. 1. Structure of Tsai-type 1/1 ACs. The polyhedra were plotted using the refined structure model of $\mathrm{GdCd}_{6}$. (a) Tsai-type concentric polyhedral shells. From left to right: disordered tetrahedron (presented as a cuboctahedron), dodecahedron, icosahedron, icosidodecahedron, and rhombic triacontahedron. (b) Body-centered periodic arrangement of $\mathrm{RE}$ icosahedra. Note that $\mathrm{RE}=\mathrm{Gd}$ in the present case. (c) The interstitial distorted octahedral picture. The length of edges (bonds) shared with the icosahedra is $5.7515 \AA$, while that of the other edges is $5.7271 \AA$.

The effect of the introduction of defects and/or impurities can be very informative to characterize condensed-matter systems. Here we report the discovery of an abrupt change in the physical property of $\left(\mathrm{Gd}_{1-x} \mathrm{Y}_{x}\right) \mathrm{Cd}_{6} 1 / 1 \mathrm{AC}$ by diluting Gd only by a few percent from the pure $\operatorname{GdCd}_{6}(x=0)$. In general, magnetic dilution of this order does not cause a significant change in ordinary magnetic systems. This suggests that $x=0$ is a "singular" point in the present system. The observed significant dilution behavior might be reflecting the uniqueness of the present system having a peculiar shortrange magnetic ordering behavior and the unique magnetic geometrical network.

\section{EXPERIMENTAL METHODS}

Samples were synthesized using the self-flux method. We used starting chemicals from Chempur, granules of $3 \mathrm{~N}$ (99.9 atom \% purity)-Y, 4N-Gd, and $6 \mathrm{~N}-\mathrm{Cd}$. The starting nominal compositions were $\left(\mathrm{Gd}_{1-x} \mathrm{Y}_{x}\right)_{5} \mathrm{Cd}_{95}(x=0$, $0.02,0.05,0.1,0.3,0.5)$ for the $1 / 1 \mathrm{ACs}$, while they were $\mathrm{Gd}_{0.8} \mathrm{Cd}_{99.2}$ and $\left(\mathrm{Gd}_{1-x} \mathrm{Y}_{x}\right)_{0.6} \mathrm{Cd}_{99.4}(x=0.1,0.3,0.5)$ for the QCs [27]. The starting materials were put in an alumina crucible and sealed in stainless steel ampules under an inert Ar atmosphere. The ampules were first heated up to $700^{\circ} \mathrm{C}$ in $10 \mathrm{~h}$ and kept for another $10 \mathrm{~h}$ to obtain homogeneous melts. Then the temperature was slowly cooled at a rate of $2{ }^{\circ} \mathrm{C} / \mathrm{h}$ from $650{ }^{\circ} \mathrm{C}$ to $500^{\circ} \mathrm{C}$ for the $1 / 1 \mathrm{AC}$ and at a rate of $1{ }^{\circ} \mathrm{C} / \mathrm{h}$ from $455^{\circ} \mathrm{C}$ to $355^{\circ} \mathrm{C}$ for the QC. Reactions were terminated by isothermally centrifuging off excess melt. Sample characterization was performed with powder x-ray diffraction (PXRD) with $\mathrm{Cu} K \alpha$ radiation, scanning electron microscopy (SEM) coupled with energy-dispersive X-ray spectroscopy (EDX), and inductively coupled plasma (ICP) mass spectrometry. Room-temperature single-crystal x-ray diffraction measurement was performed for our $\mathrm{GdCd}_{6}$ sample, and the reported structure [8] was reproduced. The refined structure is presented in Figs. 1(a)-1(c). Note that the two different
Gd-Gd distances for icosahedra [see Fig. 1(b)] and octahedra [see Fig. 1(c)] do not significantly differ, and thus the geometrical frustration on the icosahedra and octahedra can reasonably be assumed. The ICP analysis was done by Mikrolab Kolbe (Germany). The specific heat measurements were performed using a Bluefors dilution refrigerator equipped with a superconducting magnet. The specific heat data was collected using a differential membrane-based nanocalorimeter [28] down to 100-200 $\mathrm{mK}$. The sample size for specific heat measurements was approximately $(40-50)^{2} \times(10-15) \mu \mathrm{m}^{3}$ for $x=0,0.012,0.072,0.26$ and $(\sim 30)^{2} \times(5-10) \mu \mathrm{m}^{3}$ for $x=0.032$. The sample weight could not be measured due to the small sample sizes. See the Supplemental Material [25] for the conversion of the values in molar unit. The electrical resistivity was measured using the conventional four-probe method in the dilution refrigerator for $x=0,0.012$ and in a Physical Property Measurement System (PPMS) from Quantum Design Inc. for $x=0.032,0.072,0.26$, and 0.45 . For the dc magnetization and ac magnetic susceptibility measurements, we used an MPMS XL SQUID magnetometer (from Quantum Design Inc.). In this study, we used polycrystalline samples for the physical property measurements.

\section{RESULTS AND DISCUSSION}

In the following, we study the diluted systems $\left(\operatorname{Gd}_{1-x} \mathrm{Y}_{x}\right) \mathrm{Cd}_{6}$. Note that we use this formula unit (f.u.) for the AC systems. We confirmed the single phases from the powder-X-ray diffraction (PXRD) patterns for the $1 / 1 \mathrm{AC}$ samples [see Fig. 2(a)]. The lattice parameters determined from the PXRD patterns exhibit a systematic change against $x$ [see the inset of Fig. 2(a)]. We have analyzed the concentration of $\mathrm{Y}$ in the samples using inductively coupled plasma (ICP) mass spectrometry for $x=0.012$ and 0.032 and energy-dispersive $\mathrm{x}$-ray spectroscopy (EDX) for $x=0.072,0.26$, and 0.45 . In Fig. 2(b), the analyzed values of $x$ are plotted against the nominal values of the starting compositions in syntheses. All analyzed values are smaller than the nominal ones. The analysis results indicate that the $x=0.012$ and $x=0.032$ samples contain $\sim 1 \%$ and $\sim 3 \%$ of $\mathrm{Y}$ atoms on the rare-earth ( $\mathrm{Gd}$ and $\mathrm{Y})$ sites. Note that, for the QC system, the starting nominal compositions $(x=0.1,0.3$, and 0.5$)$ turned out to be $\left(\operatorname{Gd}_{1-x} \mathrm{Y}_{x}\right) \mathrm{Cd}_{7.5}(x=0.068,0.27$, and 0.47 ), respectively, from the EDX analysis. We use this formula for the QC system. We use the analyzed composition throughout this paper. See the Supplemental Material [25] for the PXRD patterns of the QC system.

Figure 3 shows the temperature dependence of electrical resistivity. We observe an inflection at $T_{\mathrm{s}}$ (plotted in the inset of Fig. 3) for all samples studied here. This reflects the structural phase transition in terms of the orientational ordering of the internal tetrahedral unit [24], which results in a monoclinic structure (in the low-temperature phase) from the cubic structure (in the high-temperature phase) [29]. Note that we determine the structural-phase-transition temperature $T_{\mathrm{s}}$ (for orientational ordering of the tetrahedral units) from peaks in the second derivative of electrical resistivity (see the Supplemental Material [25]). This indicates that there is no significant change in the structural property at low temperatures induced by the dilution. The increase in resistivity below 

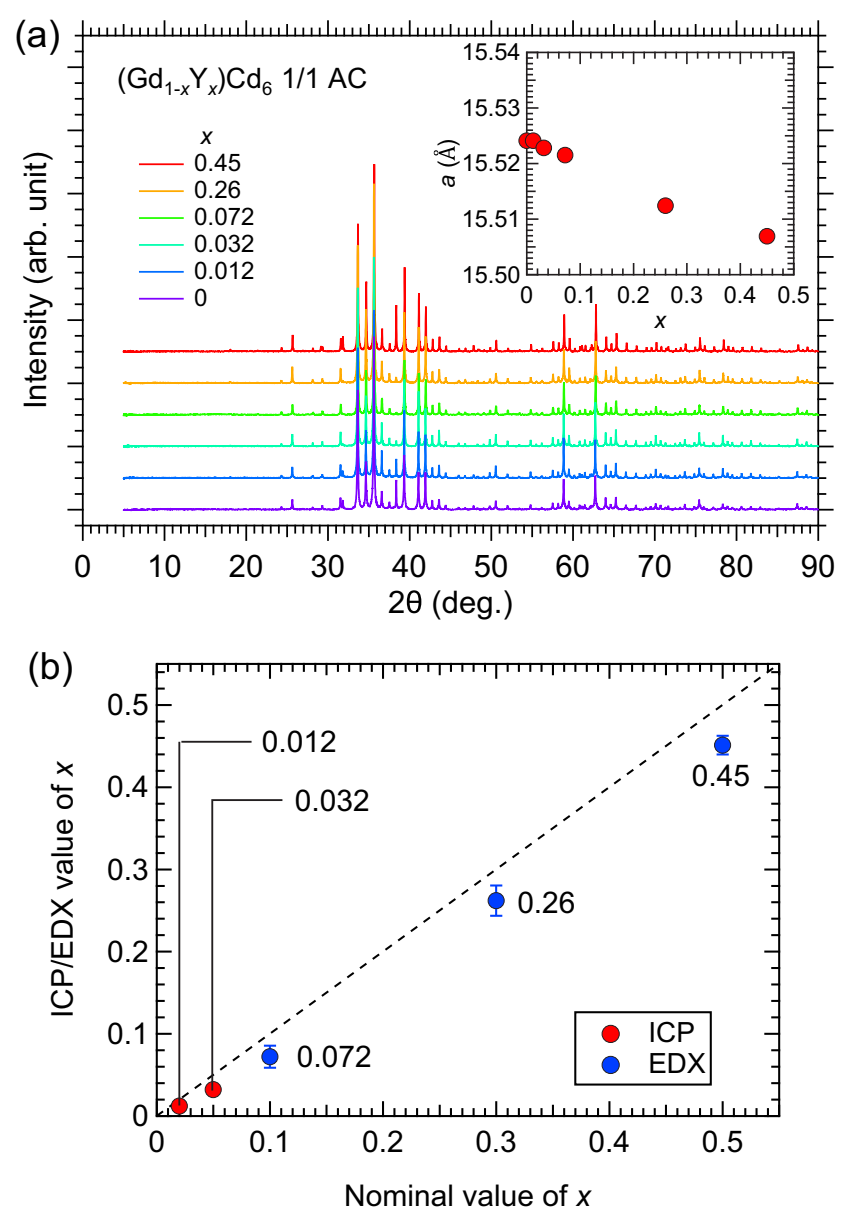

FIG. 2. Sample characterization for $\left(\operatorname{Gd}_{1-x} \mathrm{Y}_{x}\right) \mathrm{Cd}_{6} 1 / 1 \mathrm{AC}$. (a) The PXRD patterns. The inset is the lattice constant calculated from the PXRD data. Diffraction background and Bragg peaks from $\mathrm{Cu} K \alpha_{2}$ are subtracted for the plots. (b) The value of $x$ obtained from ICP and EDX analysis (plotted against the nominal value of $x$ ). The data points for the nominal $x=0.012$ and 0.032 were obtained from an ICP analysis, while those for $x=0.072,0.26$, and 0.45 were obtained from an EDX analysis. The error bars for the ICP data points are smaller than the symbol.

$\sim 40 \mathrm{~K}$ could be reflecting the possible short-range magnetic ordering/correlation in the present system [24]. Note that the increase of resistivity terminates at $T_{\mathrm{r}}^{*}$ reflecting the static magnetic ordering (shown later), below which the resistivity starts to decrease.

Figure 4(a) shows the temperature dependence of specific heat $(C)$ divided by temperature for $x=0,0.012,0.032$, 0.072 , and 0.26. All samples studied here exhibit peaks (denoted by $T_{\mathrm{A}}$ ) at $T=10-20 \mathrm{~K}$ [see the inset of Fig. 4(a)]. For $x=0$ and 0.012 , we observe secondary peaks (denoted by $T_{\mathrm{B}}$ ) below $T_{\mathrm{A}}$. We estimate the magnetic contribution to the specific heat $\left(C_{\mathrm{mag}}\right)$ by subtracting the phonon and electronic contributions. Note that we assume the Debye model for the phonon contribution above $T \sim 60 \mathrm{~K}$ and use the electronic specific heat coefficient of $\mathrm{YCd}_{6}(\gamma \approx 9 \mathrm{~mJ} / \mathrm{K}$ mol [24]). In Fig. 4(b), we plot the estimated magnetic specific heat divided by temperature $\left(C_{\mathrm{mag}} / T\right)$ for $x=0,0.012$, and 0.032 . From the $C_{\mathrm{mag}} / T$ curve, we calculate the magnetic entropy above

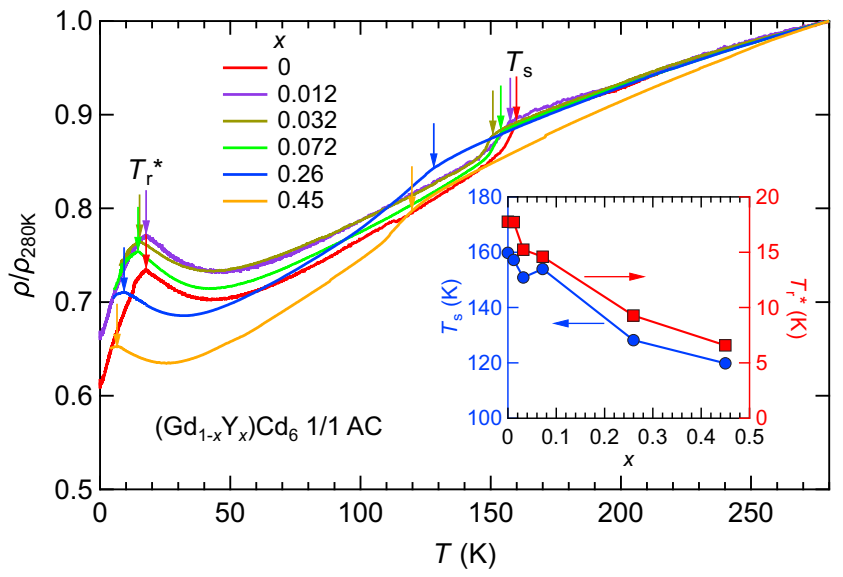

FIG. 3. Temperature dependence of electrical resistivity normalized by the value at $280 \mathrm{~K}$. The inset shows the $x$ dependence of the characteristic temperatures $T_{\mathrm{s}}$ and $T_{\mathrm{r}}^{*}$, both of which are defined from the electrical resistivity data as indicated by the arrows. The temperature $T_{\mathrm{s}}$ is defined at the inflection point in the resistivity around $120-160 \mathrm{~K}$, which reflects the orientational ordering of the tetrahedral unit. The temperature $T_{\mathrm{r}}^{*}$ is defined at the peak position in the resistivity curve below $20 \mathrm{~K}$, which seems to be reflecting the magnetic ordering.

$0.2 \mathrm{~K}$ [see the inset of Fig. 4(b)], i.e., $\Delta S_{\mathrm{mag}}(T)=S_{\mathrm{mag}}(T)-$ $S_{\text {mag }}(0.2 \mathrm{~K})=\int_{0.2 \mathrm{~K}}^{T} C_{\mathrm{mag}} / T d T$. Since $S_{\mathrm{mag}}(0.2 \mathrm{~K})$ should be negligible, one can assume $\Delta S_{\mathrm{mag}}(T) \approx S_{\text {mag }}(T)$. The magnetic entropy $\Delta S_{\text {mag }}(T)$ seems to saturate near $R \ln 8$ (where $R$ is the gas constant) above $\sim 40 \mathrm{~K}$, in agreement with the $\mathrm{Gd}^{3+}$ magnetism: note that a free $\mathrm{Gd}^{3+}$ ion has the total angular momentum of $J=S=7 / 2$ and thus it is $8(=2 J+1)$ fold degenerate. The gradual increase in $C_{\mathrm{mag}} / T$ from $\sim 40 \mathrm{~K}$ down to $T_{\mathrm{A}}$ may be reflecting the development of the short-range ordering as suggested previously [24]. Thus it is reasonable to consider that all the observed anomalies below $\sim 40 \mathrm{~K}$ should be attributed to the magnetic behavior of the present system. Note that approximately $40 \%$ of the full magnetic entropy derives from the short-range ordering developing in the temperature region of $T_{\mathrm{A}}<T \lesssim 40 \mathrm{~K}$. The relatively large deviation from $R \ln 8$ above $\sim 40 \mathrm{~K}$ for $x=0.032$ should be ascribed to the relatively smaller size of the sample used for the measurement, whose signal becomes comparable to the background signal of a calorimeter. We observe a significant qualitative difference between $x=0$ and $x \geqslant 0.012$ at low temperatures (in the ordered phase below $T_{\mathrm{A}}$ ), indicating that the magnetic properties of the present pure system $(x=0)$ are sensitive to defects in the magnetic sites. At low temperatures, the pure system $(x=0)$ exhibits a significant upturn below $\sim 4 \mathrm{~K}$ followed by two inflections (at $T_{\mathrm{C}}$ and $T_{\mathrm{D}}$ ), which are absent for $x \geqslant 0.012$. Instead of the double-inflection structure, the diluted systems exhibit a single broad peak (for $x=0.012$ and 0.032) or a shoulderlike structure (for $x=0.072$ and $0.26)$. These are denoted by the onset points $\left(T_{\mathrm{sh}}^{*}\right)$ below which $C / T$ decreases again. We notice a difference in the shape of $C / T$ between our result and that of Mori et al. for $x=0$ : this fact may indicate that the magnetic property of the pure system $(x=0)$ is very sensitive to small differences in the samples (homogeneity, defects, and so on), as demonstrated 

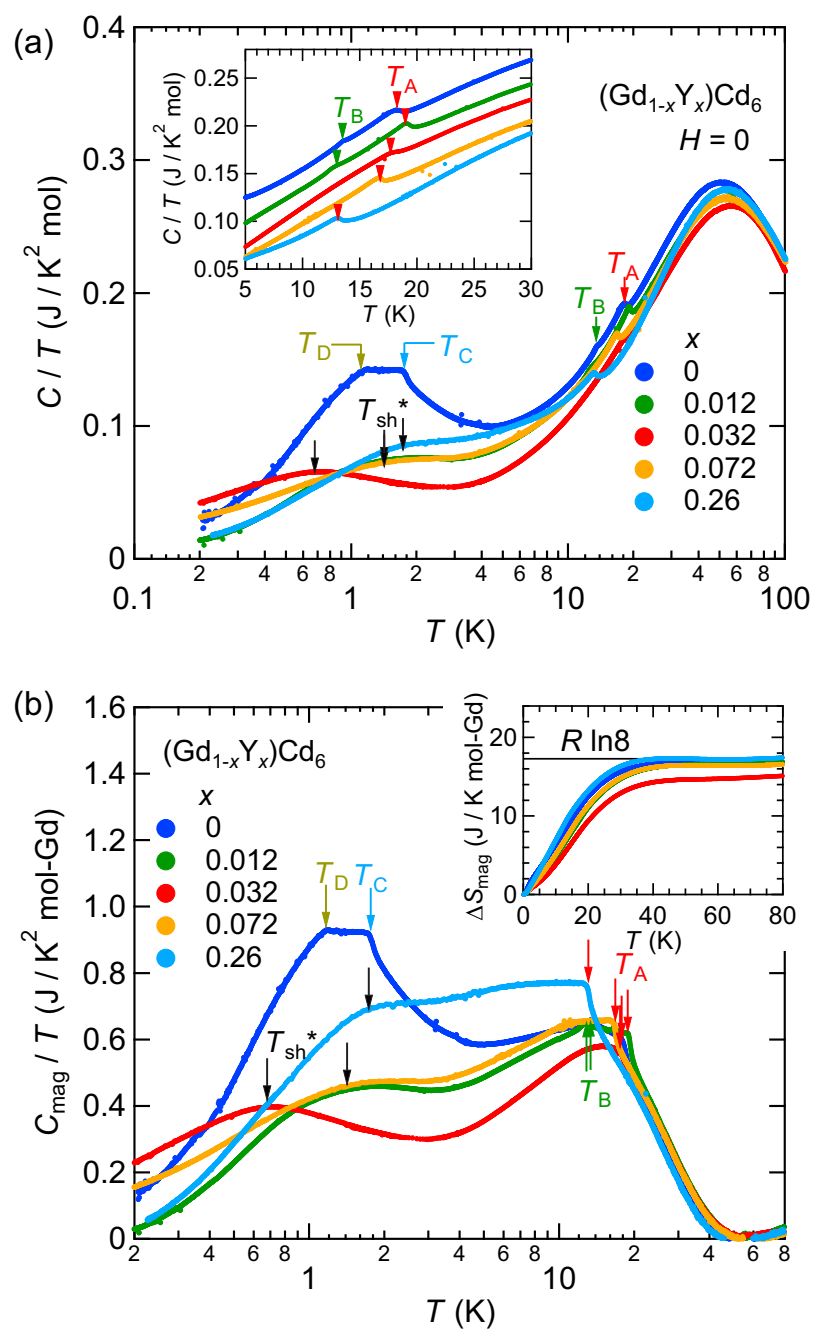

FIG. 4. Thermodynamic property of $\left(\operatorname{Gd}_{1-x} \mathrm{Y}_{x}\right) \mathrm{Cd}_{6} 1 / 1$ AC. (a) Temperature dependence of specific heat divided by temperature $(C / T)$. We define the characteristic temperatures $T_{\mathrm{A}}, T_{\mathrm{B}}, T_{\mathrm{C}}, T_{\mathrm{D}}$, and $T_{\mathrm{sh}}^{*}$ as indicated by the arrows. Note that $T_{\mathrm{C}}$ and $T_{\mathrm{D}}$ are defined only in $x=0$, while $T_{\mathrm{sh}}^{*}$ defined for $x \geqslant 0.012$ indicates the onset below which $C / T$ decreases further. The inset shows the close-up view of the region $5 \leqslant T \leqslant 30 \mathrm{~K}$ : note that the data is shifted in the vertical direction for the visibility of the anomalies at $T_{\mathrm{A}}$ and $T_{\mathrm{B}}$ indicated by the triangles. (b) Temperature dependence of the magnetic specific heat divided by temperature $\left(C_{\mathrm{mag}} / T\right)$ for $x=0,0.012$, and 0.032 . The inset shows the temperature dependence of the magnetic entropy $\Delta S_{\text {mag }}$.

here by introducing defects in the magnetic sites intentionally. See the Supplemental Material [25] for more detailed information about the specific heat results.

Figure 5(a) shows the temperature dependence of the inverse magnetic susceptibility $1 / \chi_{4 f}$ where $\chi_{4 f} \equiv \chi-\chi_{0}$. Note that $\chi=M / H$ is the susceptibility measured at $H=5$ kOe and $\chi_{0}$ is a temperature-independent non- $4 f$-electron term. We observe linear behavior at $T \gtrsim 80 \mathrm{~K}$, indicating Curie-Weiss behavior $\chi_{4 f}=C_{\mathrm{CW}} /\left(T-\theta_{\mathrm{CW}}\right)$ (where $C_{\mathrm{CW}}$ is a constant) with the negative values of Curie-Weiss temperature $\theta_{\mathrm{CW}}$. The deviation from the linearity below $\sim 80 \mathrm{~K}$ may be attributed to the effect of short-range ordering. The effective
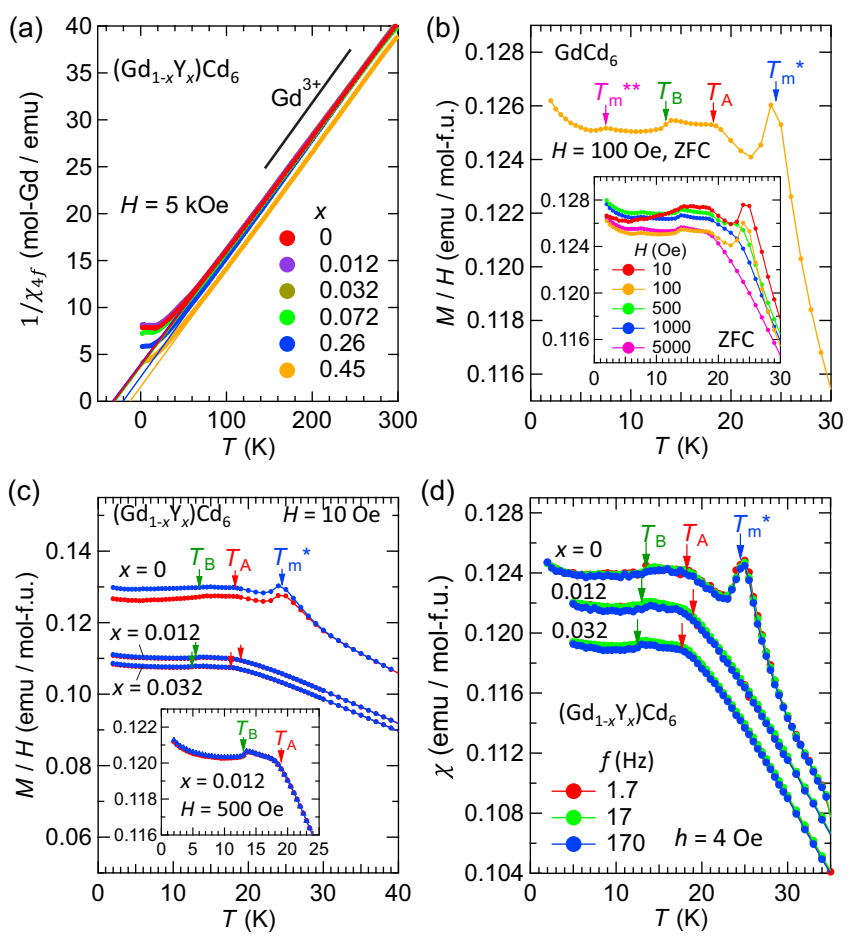

FIG. 5. Magnetic property of $\left(\operatorname{Gd}_{1-x} \mathrm{Y}_{x}\right) \mathrm{Cd}_{6} 1 / 1$ AC. (a) Temperature dependence of $1 / \chi_{4 f}$. The solid lines superposed on the data curves indicate the linear fitting results for the experimental curves in the temperature interval of $100 \lesssim T \lesssim 280 \mathrm{~K}$. The black solid line is a guide for the theoretical values of effective magnetic moment of $\mathrm{Gd}^{3+}: p_{\text {eff }}=7.94 \mu_{\mathrm{B}} / \mathrm{Gd}$. (b) Temperature dependence of magnetization (plotted as $M / H$ ) with $H=100$ Oe for the pure system $(x=0)$. The inset shows the magnetization curves for various values of magnetic field. We define the characteristic temperatures $T_{\mathrm{m}}^{*}$ and $T_{\mathrm{m}}^{* *}$ as indicated by the arrows along with $T_{\mathrm{A}}$ and $T_{\mathrm{B}}$ (defined from the specific heat). (c) Temperature dependence of the magnetization at $H=10$ Oe for $x=0,0.012$, and 0.032 . The red and blue curves indicate ZFC and FC magnetization curves, respectively. The inset shows the magnetization for $x=0.012$ with $H=500 \mathrm{Oe}$. There is no anomaly corresponding to $T_{\mathrm{m}}^{*}$ and $T_{\mathrm{m}}^{* *}$ in $x=0.012$ and $x=0.032$. (d) ac magnetic susceptibility for $x=0,0.012$, and 0.032 . The amplitude of the applied excitation magnetic field is set to $h=4$ Oe.

magnetic moment estimated from the slope is close to the theoretical value of free $\mathrm{Gd}^{3+}$ ion $\left(7.94 \mu_{\mathrm{B}} / \mathrm{Gd}\right)$ : the maximum deviation is $\sim 3 \%$ in this analysis. See the Supplemental Material [25] for details about the analysis of Curie-Weiss behavior. We do not observe any inflection at $T_{\mathrm{s}}$, which was observed previously by Mori et al. [24] probably because the contribution of the structural transition to the magnetic susceptibility is very weak. Note that Mori et al. do not observe this anomaly for other $\mathrm{RECd}_{6}$ ACs [24]. Figure 5(b) shows the zero-field-cooling (ZFC) magnetization curves (plotted as $M / H)$ of $\mathrm{GdCd}_{6}$ with $H=100 \mathrm{Oe}$. We observe two anomalies at $T_{\mathrm{A}}$ and $T_{\mathrm{B}}$ confirming the magnetic origin, which are defined from the specific heat data, and observe two additional notable temperature points denoted as $T_{\mathrm{m}}^{*}$ and $T_{\mathrm{m}}^{* *}$, which are absent from the specific heat. We speculate that $T_{\mathrm{m}}^{*}$ reflects the magnetic state precursor to the static magnetic ordering 
established at $T_{\mathrm{A}}$, while $T_{\mathrm{m}}^{* *}$ is associated with a slight change in the magnetic configuration (see also below). Note that $T_{\mathrm{A}}, T_{\mathrm{B}}$, and $T_{\mathrm{m}}^{* *}$ have been noticed previously in Ref. [24]. The presence of $T_{\mathrm{m}}^{*}$ and $T_{\mathrm{m}}^{* *}$ distinguishes the pure system $(x=0)$ from the slightly diluted ones $(x=0.012$ and 0.032$)$, which do not show anomalies at $T_{\mathrm{m}}^{*}$ nor $T_{\mathrm{m}}^{* *}$, as shown in Fig. 5(c) and the inset therein, whereas the ones at $T_{\mathrm{A}}$ and $T_{\mathrm{B}}$ remain. We observe a bifurcation below $T_{\mathrm{m}}^{*}$ in the $\mathrm{ZFC}$ and field-cooling (FC) magnetization curves of $x=0$ with $H=10 \mathrm{Oe}$, which is absent for $x=0.012$ and 0.032 . We note that there is another qualitative difference between the slightly diluted systems $(x=0.012$ and 0.032$)$ and the diluted systems $(x \geqslant 0.072)$ : in the $x \geqslant 0.072$ systems, an inflection in magnetization curve was observed at $T_{\mathrm{A}}$, which is followed by a ZFC-FC bifurcation, while there is no secondary anomaly corresponding to $T_{\mathrm{B}}$. For all the pure and diluted $\mathrm{AC}$ systems studied here, we do not observe a frequency dependence in the ac magnetic susceptibility [see Fig. 5(d) for $x=0,0.012$, and 0.032], indicating a static type of magnetic ordering below the transitions. See the Supplemental Material [25] for the magnetic data for $x=0.072,0.26$, and 0.45 .

For clarity, we plot the above-mentioned characteristic temperatures against $x$ in Fig. 6(a). In Fig. 6(b), we plot the value of $\left|\theta_{\mathrm{CW}}\right| / T_{\mathrm{mag}}$, where $T_{\mathrm{mag}}$ is a magnetic ordering temperature. In general, the value of $\left|\theta_{\mathrm{CW}}\right| / T_{\text {mag }}$ reflects the degree of magnetic frustration [30]. In this study, we assume $T_{\text {mag }}=T_{\mathrm{A}}$. Although there is a structural phase transition with respect to the orientational ordering of internal tetrahedra in the present $\mathrm{AC}$ systems, the value of $\left|\theta_{\mathrm{CW}}\right|$ is close to that of $T_{\mathrm{mag}}\left(=T_{\mathrm{A}}\right)$, which is reasonable considering the static magnetic ordering, and both $\left|\theta_{\mathrm{CW}}\right|$ and $T_{\mathrm{mag}}$ exhibit similar $x$ dependence. Thus the estimated value of $\left|\theta_{\mathrm{CW}}\right| / T_{\mathrm{mag}}$ shall be a relevant measure of the degree of frustration for the present system. The value of $\left|\theta_{\mathrm{CW}}\right| / T_{\mathrm{mag}}$ is slightly larger than 1 , which indicates there is a slight magnetic frustration. The overall behavior of the $\left|\theta_{\mathrm{CW}}\right| / T_{\mathrm{mag}}$ value is to decrease as $x$ increases for $x>0.1$ [see Fig. 6(b)] despite the introduction of chemical disorder, suggesting that the pure and slightly diluted systems $(x \lesssim 0.1)$ should be considered as a staticorder system with slight frustration. We conjecture that the relatively large values of $\left|\theta_{\mathrm{CW}}\right| / T_{\mathrm{mag}}$ in the ternary Gd-based ACs (see the Supplemental Material [25]) are due to the randomness caused by the inherent mixed sites. The anomaly at $T_{\mathrm{m}}^{*}\left(\psi \theta_{\mathrm{CW}} \mid\right)$ in the pure system $(x=0)$ appears to reflect a precursor of the magnetically ordered state. This precursorlike anomaly at $T_{\mathrm{m}}^{*}$ is easily destroyed by a relatively large magnetic field of $H \geqslant 500 \mathrm{Oe}$ [see the inset of Fig. 5(b)]. We speculate that reconfigurations of the magnetic order occur at the other characteristic temperatures (e.g., $T_{\mathrm{B}}$ and $T_{\mathrm{m}}^{* *}$ ), most of which are suppressed by a dilution.

It was reported previously that the short-range magnetic ordering starts to develop at $T \sim 40 \mathrm{~K}$ (higher than $T_{\mathrm{mag}}$ ) in the pure system $(x=0)$ [24]. In this study, we find that the dilute systems $(x=0.012,0.032,0.072,0.26$, and 0.45$)$ also have such short-range ordering developing at $T>T_{\mathrm{mag}}$, as suggested by the increase in resistivity below $\sim 40 \mathrm{~K}$ (see Fig. 3), the magnetic specific heat developing at $T>T_{\text {mag }}$ [see Fig. 4(b)], and the deviation of the magnetic susceptibility from Curie-Weiss law below $\sim 80 \mathrm{~K}$ [see Fig. 5(a)]. This finding indicates that the short-range ordering is inherent to
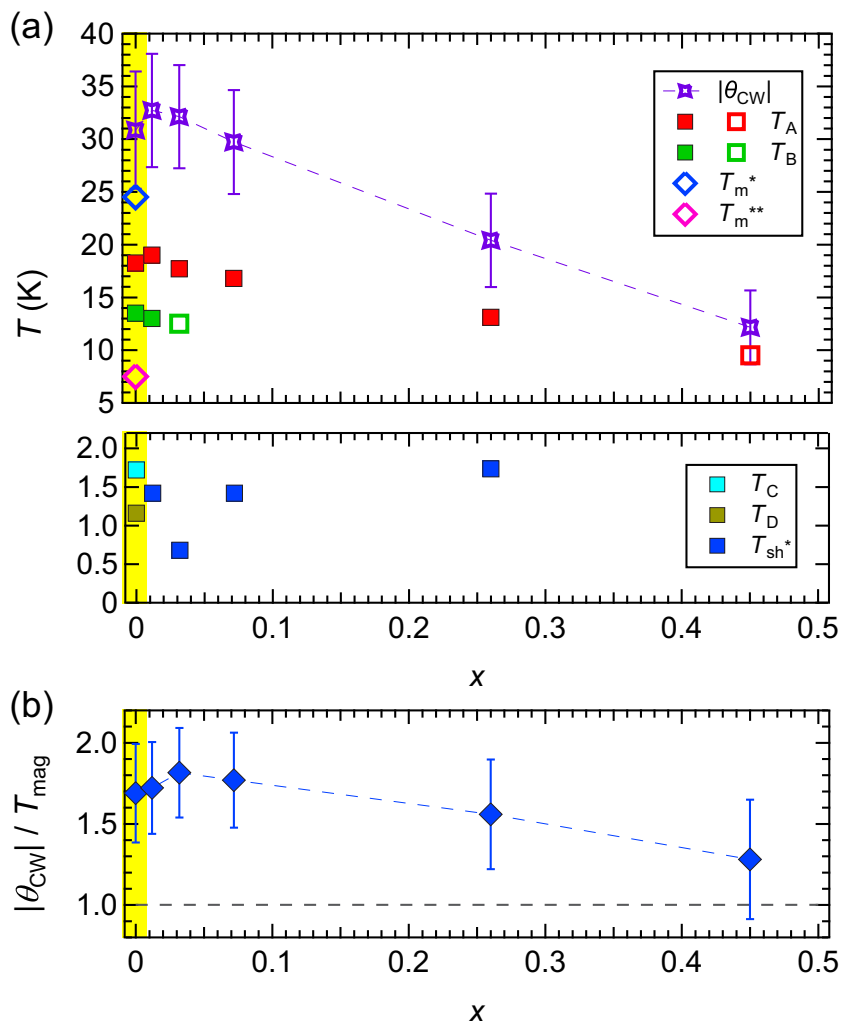

FIG. 6. $T-x$ map for the characteristic temperature points and the $x$ dependence of the magnitude of magnetic frustration for $\left(\mathrm{Gd}_{1-x} \mathrm{Y}_{x}\right) \mathrm{Cd}_{6} 1 / 1$ AC. (a) The plot of the characteristic temperatures obtained from the specific heat (filled symbols) and magnetization (open symbols) data. (b) The plot of Curie-Weiss temperature $\left(\left|\theta_{\mathrm{CW}}\right|\right)$ divided by magnetic ordering temperature $\left(T_{\text {mag }}=T_{\mathrm{A}}\right)$, suggesting the degree of frustration. Note: for $T_{\mathrm{A}}$ and $T_{\mathrm{B}}$, the open symbols are from the magnetic data, while the filled symbols are from both magnetic and specific-heat data. The color background (for $x=0$ ) is to highlight the "singular" magnetic point suggested in this paper.

the present AC system regardless of the degree of dilution for $x \lesssim 0.5$, although the onset temperature of the short-range ordering (the onset of the upturn in resistivity) seems to decrease gradually as $x$ increases (see Fig. 3).

We note that the corresponding QC system, $\left(\operatorname{Gd}_{1-x} \mathrm{Y}_{x}\right) \mathrm{Cd}_{7.5}$, does not exhibit such a qualitative change against substitution. Figure 7(a) shows the temperature dependence of $1 / \chi_{4 f}$ of $\left(\operatorname{Gd}_{1-x} \mathrm{Y}_{x}\right) \mathrm{Cd}_{7.5}$ QC. We observe a good linear behavior of $1 / \chi_{4 f}$ (even down to just above the freezing temperature $T_{\mathrm{f}}$ ), indicating that the magnetic susceptibility exhibits Curie-Weiss law. This also suggests that there is no short-range ordering developing above $T_{\mathrm{f}}$ for the QCs, unlike the ACs. Note that we use the same experimental data for the magnetization of the pure system $(x=0)$ in Ref. [31], but we reanalyzed it here independently. Figure 7(b) shows the ZFC-FC magnetization (plotted as $M / H$ ) of the QCs under a small magnetic field of $H=10$ Oe. The pure system $(x=0)$ has been known to exhibit a SG behavior suggested from the memory effect [31]. The diluted QC also exhibits the memory effect and thus a SG behavior (see the Supplemental Material [25]). This SG behavior in the present QC system might be due to intrinsic chemical disorder, since approximately 

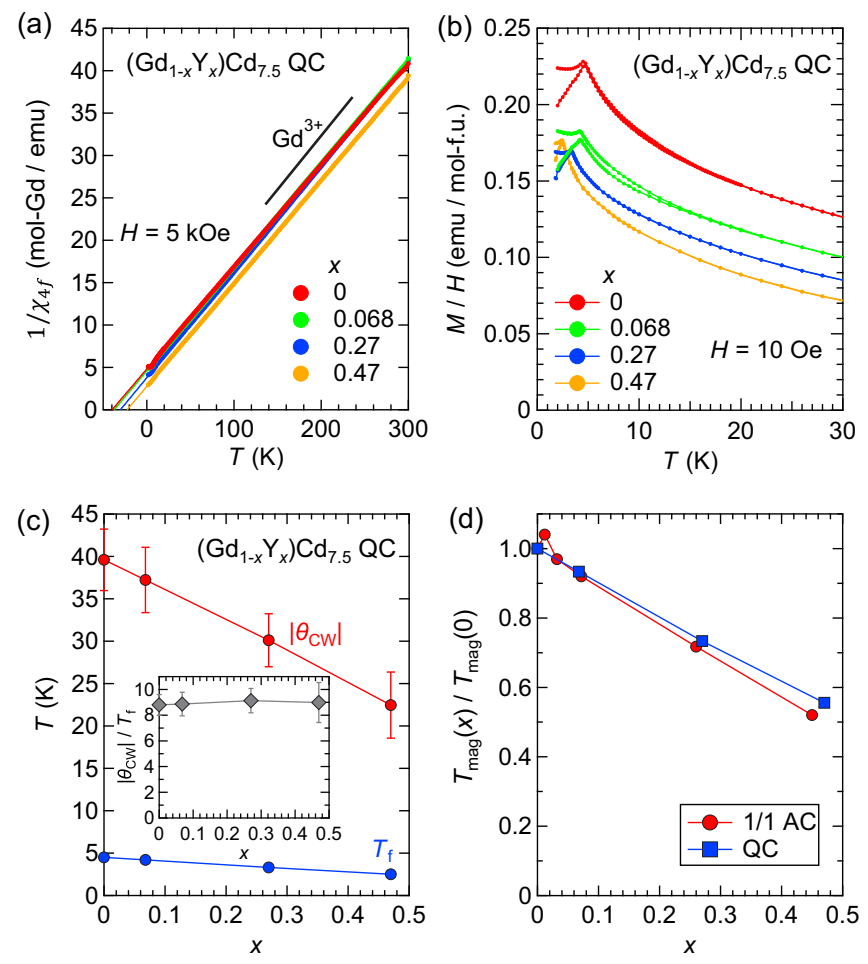

FIG. 7. (a) Temperature dependence of $1 / \chi_{4 f}$ for the $\left(\operatorname{Gd}_{1-x} \mathrm{Y}_{x}\right) \mathrm{Cd}_{7.5}$ QCs. The solid lines superposed on the data curves indicate the linear fitting results for the experimental curves in the temperature interval of $100 \lesssim T \lesssim 280 \mathrm{~K}$. The black solid line is the guide for the theoretical values of effective magnetic moment for $\mathrm{Gd}^{3+}: p_{\text {eff }}=7.94 \mu_{\mathrm{B}} / \mathrm{Gd}$. (b) Temperature dependence of the ZFC-FC magnetization measured with $H=10$ Oe. (c) The variation of Curie-Weiss temperature $\left(\left|\theta_{\mathrm{CW}}\right|\right)$ and freezing temperatures $\left(T_{\mathrm{f}}\right)$ against the composition $x$ for the $\left(\mathrm{Gd}_{1-x} \mathrm{Y}_{x}\right) \mathrm{Cd}_{7.5}$ QCs. The inset shows the degree of frustration $\left|\theta_{\mathrm{CW}}\right| / T_{\mathrm{f}}$. (d) The variation of magnetic ordering temperatures $\left(T_{\mathrm{mag}}\right)$ normalized by the value of the pure system $(x=0)$ against the composition $x$ for the $\left(\operatorname{Gd}_{1-x} \mathrm{Y}_{x}\right) \mathrm{Cd}_{6} 1 / 1$ ACs and the $\left(\operatorname{Gd}_{1-x} \mathrm{Y}_{x}\right) \operatorname{Cd}_{7.5}$ QCs. Note that $T_{\text {mag }}=T_{\mathrm{A}}$ for the $1 / 1 \mathrm{ACs}$, while $T_{\text {mag }}=T_{\mathrm{f}}$ for the QCs.

$20 \%$ of the magnetic icosahedral sites are estimated to be inherently occupied by nonmagnetic $\mathrm{Cd}$ atoms [32]. We observe monotonic shift of the freezing temperature $T_{\mathrm{f}}$ toward lower temperatures by the dilution. Note that we determine the freezing temperature $T_{\mathrm{f}}$ from the cusp (peak) point. The freezing temperature $T_{\mathrm{f}}$ and the Curie-Weiss temperature $\left|\theta_{\mathrm{CW}}\right|$ decrease monotonically by the dilution [see Fig. 7(c)], while the value of $\left|\theta_{\mathrm{CW}}\right| / T_{\mathrm{f}}=8-9$ remains almost unchanged [see the inset of Fig. 7(c)].

It is interesting to compare the ACs and QCs, which share similar local magnetic icosahedral shells yet exhibit significantly different behaviors. For the entire dilution region $(0<$ $x \lesssim 0.5$ ), the ACs exhibit static magnetic ordering (and the short-range ordering) with small values of $\left|\theta_{\mathrm{CW}}\right| / T_{\text {mag }}$ despite the presence of chemical disorder in the magnetic sites for $x>0$ [see Fig. 6(b)], while the QCs exhibit SG behavior with large values of $\left|\theta_{\mathrm{CW}}\right| / T_{\mathrm{mag}}$ [see the inset of Fig. 7(c)]. This comparison allows us to conjecture that the static ordering (and the short-range ordering) behavior of the ACs stems from the periodicity and thus the octahedral magnetic network plays an important role, while the SG behavior of the QC derives not only from the inherent chemical disorder in the icosahedral shell [32] but also from the aperiodicity. On the other hand, the overall $x$ dependence of $T_{\text {mag }}(x) / T_{\text {mag }}(0)$ (i.e., the contribution of the dilution to the energy scale of magnetic ordering/interaction) seems to be universal, as it appears for both the AC and QC [see Fig. 7(d)], and thus likely is determined from the local icosahedral magnetic cluster shared by the AC and QC.

Regarding the significant dilution behavior observed in the present system, we speculate that both the short-range correlation/ordering and the slight magnetic frustration are important. The former seems to be a local effect inherent in the present AC system as it does not differ significantly by the dilution for $x \lesssim 0.5$. The short-range correlation developing at $T>T_{\text {mag }}$ is reminiscent of spin-glass QCs $\mathrm{Zn}-\mathrm{Mg}-\mathrm{Tb}$ [3] and $\mathrm{Zn}-\mathrm{Fe}-\mathrm{Sc}$ [33]. These systems exhibit peculiar shortrange correlation behavior (which seems to occur in the cluster scale) whose onset temperatures ( $T \sim 50 \mathrm{~K}$ for $\mathrm{Zn}$ $\mathrm{Mg}-\mathrm{Tb}$ and $T \sim 200 \mathrm{~K}$ for $\mathrm{Zn}-\mathrm{Fe}-\mathrm{Sc}$ ) are well above their freezing temperatures $\left(T_{\mathrm{f}} \approx 5.8 \mathrm{~K}\right.$ for $\mathrm{Zn}-\mathrm{Mg}-\mathrm{Tb}$ and $T_{\mathrm{f}} \approx$ $7 \mathrm{~K}$ for $\mathrm{Zn}-\mathrm{Fe}-\mathrm{Sc}$ ). The present system may also have a similar short-range correlation mechanism with these QCs, although its origin remains to be clarified. The slight magnetic frustration $\left(\left|\theta_{\mathrm{CW}}\right| / T_{\mathrm{f}} \sim 1.7\right)$ is not as significant as those in the other typical frustrated systems including the present spin-glass QC system $\left(\left|\theta_{\mathrm{CW}}\right| / T_{\mathrm{f}}=8-9\right)$. This weak frustration of the present $\mathrm{AC}$ system might be mainly due to the following two reasons. First, there is no intrinsic chemical disorder in $\mathrm{GdCd}_{6}$ unlike the other ternary $\mathrm{AC}$ compounds, and thus there should be no magnetic frustration caused by the randomness through the RKKY interaction. Second, the structural transition (from the high-temperature cubic phase to the low-temperature monoclinic phase) [29] would lower the symmetry of the structure which might affect the degree of geometrical frustration. Note that the distortion of structures will not prevent possible effects of geometrical frustration as geometrical frustration seems to be effective even in distorted frustrated systems $[34,35]$. It remains to be clarified whether the origin of the weak frustration is geometrical, although we conjecture that it has a geometrical origin as the value of $\left|\theta_{\mathrm{CW}}\right| / T_{\mathrm{f}}$ decreases as the $\mathrm{Y}$ concentration $x$ increases in spite of the increase in chemical disorder. It seems to be difficult to understand the weak frustration considering only the long-range oscillating nature of the RKKY interaction. Such weak frustration itself would not generally trigger the remarkable change by the magnetic dilution by only a few percentages, yet the coexistence of the weak frustration and the short-range correlation may induce such peculiar magnetic dilution behavior. Note that this behavior may not solely be due to the short-range correlation, as there is no qualitative difference between the largely diluted systems $(x=0.26$ and $x=0.45$ ) having slightly less magnetic frustration despite the presence of chemical disorder. We also note that the magnetic frustration in the present system should be distinguished from the conventional frustrated magnets such as two-dimensional kagome (with the number of nearest-neighbor magnetic sites: $z=4)$ and three-dimensional pyrochlore $(z=6)$ systems, because the present $\mathrm{GdCd}_{6}$ system $(z=8$ by considering the octahedral network) basically exhibits the static magnetic 
order with a slight frustration value $\left|\theta_{\mathrm{CW}}\right| / T_{\mathrm{f}} \sim 1.7$. This is in line with the general tendency that a larger $z$ value (which lowers the percolation concentration of systems) makes it more probable for long-range ordering to occur [36]. However, at present, the exact nature of the magnetic state of this system is unknown; the resistivity starts to drop at $T_{\mathrm{A}}$, suggesting that the system is magnetically long-range ordered below $T_{\mathrm{A}}$. Note that techniques such as neutron scattering experiments may not be employed, since both $\mathrm{Gd}$ and $\mathrm{Cd}$ are good neutron absorption sources.

\section{CONCLUSION}

In conclusion, we have discovered an unusual magnetic behavior upon slightly diluting magnetic $\mathrm{Gd}$ by nonmagnetic $\mathrm{Y}$ in the bulk magnetic and thermodynamic quantities of $\left(\operatorname{Gd}_{1-x} \mathrm{Y}_{x}\right) \mathrm{Cd}_{6} 1 / 1 \mathrm{ACs}$, while the corresponding QC exhibits only a monotonic change of its SG behavior. We suggest that the pure system $\mathrm{GdCd}_{6}$ is at a singular magnetic point due to the short-range magnetic correlation and the slight magnetic frustration specific to the present AC system, and thus its physical property exhibits a significant change by magnetic dilution of even only a few percent. This work highlights the peculiarity of a magnetic $\mathrm{AC}$ system, which has a unique magnetic geometrical network without mixed sites (chemical disorder). This study can promote further investigation of the magnetism of QCs and ACs.

\section{ACKNOWLEDGMENTS}

We thank the Knut and Alice Wallenberg Foundation (Grant No. KAW 2018.0019), the Carl Tryggers Stiftelse för Vetenskaplig Forskning (Grant No. CTS 19:235), Stiftelsen Olle Engkvist (Grant No. 184-546), and the Swedish Research Council (VR) including Dnr 2016-04516.

T.S. and F.D. contributed equally to this work.
[1] H. Takakura, C. P. Gómez, A. Yamamoto, M. De Boissieu, and A. P. Tsai, Atomic structure of the binary icosahedral $\mathrm{Yb}-\mathrm{Cd}$ quasicrystal, Nat. Mater. 6, 58 (2007).

[2] K. Deguchi, S. Matsukawa, N. K. Sato, T. Hattori, K. Ishida, H. Takakura, and T. Ishimasa, Quantum critical state in a magnetic quasicrystal, Nat. Mater. 11, 1013 (2012).

[3] T. J. Sato, H. Takakura, A. P. Tsai, and K. Shibata, Magnetic excitations in the $\mathrm{Zn}-\mathrm{Mg}$-Tb icosahedral quasicrystal: An inelastic neutron scattering study, Phys. Rev. B 73, 054417 (2006).

[4] T. Fujiwara and Y. Ishii, Quasicrystals (Elsevier Science, Amsterdam, 2007), Vol. 3.

[5] A. P. Tsai, J. Q. Guo, E. Abe, H. Takakura, and T. J. Sato, A stable binary quasicrystal, Nature (London) 408, 537 (2000).

[6] A. I. Goldman, Magnetism in icosahedral quasicrystals: Current status and open questions, Sci. Technol. Adv. Mater. 15, 044801 (2014).

[7] A. I. Goldman and R. F. Kelton, Quasicrystals and crystalline approximants, Rev. Mod. Phys. 65, 213 (1993).

[8] C. P. Gómez and S. Lidin, Comparative structural study of the disordered $M \mathrm{Cd}_{6}$ quasicrystal approximants, Phys. Rev. B 68, 024203 (2003).

[9] N. K. Sato, S. Matsukawa, K. Nobe, K. Imura, K. Deguchi, and T. Ishimasa, Quantum critical behavior in magnetic quasicrystals and approximant crystals, J. Phys.: Conf. Ser. 868, 012005 (2017).

[10] P. Koželj, S. Jazbec, S. Vrtnik, A. Jelen, J. Dolinšek, M. Jagodič, Z. Jagličić, P. Boulet, M. C. de Weerd, J. Ledieu, J. M. Dubois, and V. Fournée, Geometrically frustrated magnetism of spins on icosahedral clusters: $\mathrm{The}_{3} \mathrm{Gd}_{3} \mathrm{Au}_{13} \mathrm{Sn}_{4}$ quasicrystalline approximant, Phys. Rev. B 88, 214202 (2013).

[11] M. G. Kim, G. Beutier, A. Kreyssig, T. Hiroto, T. Yamada, J. W. Kim, M. de Boissieu, R. Tamura, and A. I. Goldman, Antiferromagnetic order in the quasicrystal approximant $\mathrm{Cd}_{6} \mathrm{~Tb}$ studied by x-ray resonant magnetic scattering, Phys. Rev. B 85, 134442 (2012).

[12] A. Ishikawa, T. Hiroto, K. Tokiwa, T. Fujii, and R. Tamura, Composition-driven spin glass to ferromagnetic transition in the quasicrystal approximant Au-Al-Gd, Phys. Rev. B 93, 024416 (2016).
[13] T. Sawano, T. Shiino, K. Imura, K. Deguchi, S. Ohhashi, A.-P. Tsai, and N. K. Sato, Local quantum fluctuations in Kondo quasicrystal approximant Ag-In- $\left(\mathrm{Ce}_{x} \mathrm{Y}_{1-x}\right)$, J. Phys. Soc. Jpn. 89, 014703 (2020).

[14] P. Wang, Z. M. Stadnik, K. Al-Qadi, and J. Przewoźnik, A comparative study of the magnetic properties of the $1 / 1$ approximant $\mathrm{Ag}_{50} \mathrm{In}_{36} \mathrm{Gd}_{14}$ and the icosahedral quasicrystal $\mathrm{Ag}_{50} \mathrm{In}_{36} \mathrm{Gd}_{14}$, J. Phys.: Condens. Matter 21, 436007 (2009).

[15] R. Tamura, Y. Muro, T. Hiroto, K. Nishimoto, and T. Takabatake, Long-range magnetic order in the quasicrystalline approximant $\mathrm{Cd}_{6} \mathrm{~Tb}$, Phys. Rev. B 82, 220201(R) (2010).

[16] T. Hiroto, G. H. Gebresenbut, C. P. Gómez, Y. Muro, M. Isobe, Y. Ueda, K. Tokiwa, and R. Tamura, Ferromagnetism and reentrant spin-glass transition in quasicrystal approximants AuSM-Gd (SM = Si, Ge), J. Phys.: Condens. Matter 25, 426004 (2013).

[17] H. Miyazaki, T. Sugimoto, K. Morita, and T. Tohyama, Magnetic orders induced by RKKY interaction in Tsai-type quasicrystalline approximant Au-Al-Gd, Phys. Rev. Mater. 4, 024417 (2020).

[18] K. Inagaki, S. Suzuki, A. Ishikawa, T. Tsugawa, F. Aya, T. Yamada, K. Tokiwa, T. Takeuchi, and R. Tamura, Ferromagnetic 2/1 quasicrystal approximants, Phys. Rev. B 101, 180405(R) (2020).

[19] G. Gebresenbut, T. Shiino, D. Eklöf, D. C. Joshi, F. Denoel, R. Mathieu, U. Häussermann, and C. Pay Gómez, Atomic-scale tuning of Tsai-type clusters in RE-Au-Si systems $(\mathrm{RE}=\mathrm{Gd}$, Tb, Ho), Inorg. Chem. 59, 9152 (2020).

[20] T. J. Sato, A. Ishikawa, A. Sakurai, M. Hattori, M. Avdeev, and R. Tamura, Whirling spin order in the quasicrystal approximant $\mathrm{Au}_{72} \mathrm{Al}_{14} \mathrm{~Tb}_{14}$, Phys. Rev. B 100, 054417 (2019).

[21] T. Hiroto, T. J. Sato, H. Cao, T. Hawai, T. Yokoo, S. Itoh, and R. Tamura, Noncoplanar ferrimagnetism and local crystalline-electric-field anisotropy in the quasicrystal approximant $\mathrm{Au}_{70} \mathrm{Si}_{17} \mathrm{~Tb}_{13}$, J. Phys.: Condens. Matter 32, 415802 (2020).

[22] K. Imura, K. Nobe, K. Deguchi, M. Matsunami, H. Miyazaki, A. Yasui, E. Ikenaga, and N. K. Sato, First observation of heavy 
fermion behavior in Ce-based icosahedral approximant, J. Phys. Soc. Jpn. 86, 093702 (2017).

[23] S. Matsukawa, K. Deguchi, K. Imura, T. Ishimasa, and N. K. Sato, Pressure-driven quantum criticality and $\mathrm{T} / \mathrm{H}$ scaling in the icosahedral Au-Al-Yb approximant, J. Phys. Soc. Jpn. 85, 063706 (2016).

[24] A. Mori, H. Ota, S. Yoshiuchi, K. Iwakawa, Y. Taga, Y. Hirose, T. Takeuchi, E. Yamamoto, Y. Haga, F. Honda, R. Settai, and Y. Ōnuki, Electrical and magnetic properties of quasicrystal approximants $\mathrm{RCd}_{6}$ (R: rare earth), J. Phys. Soc. Jpn. 81, 024720 (2012).

[25] See Supplemental Material at http://link.aps.org/supplemental/ 10.1103/PhysRevB.104.224411 for details about synthesis conditions and additional information about the magnetic, thermodynamic, and transport properties of the $\left(\operatorname{Gd}_{1-x} \mathrm{Y}_{x}\right) \mathrm{Cd}_{6} \mathrm{AC}$ and the magnetic property of the corresponding QC.

[26] F. Labib, D. Okuyama, N. Fujita, T. Yamada, S. Ohhashi, T. J. Sato, and A.-P. Tsai, Magnetic properties of icosahedral quasicrystals and their cubic approximants in the $\mathrm{Cd}-\mathrm{Mg}-\mathrm{RE}$ $(\mathrm{RE}=\mathrm{Gd}, \mathrm{Tb}, \mathrm{Dy}, \mathrm{Ho}, \mathrm{Er}$, and Tm) systems, J. Phys.: Condens. Matter 32, 415801 (2020).

[27] A. I. Goldman, T. Kong, A. Kreyssig, A. Jesche, M. Ramazanoglu, K. W. Dennis, S. L. Bud'ko, and P. C. Canfield, A family of binary magnetic icosahedral quasicrystals based on rare earths and cadmium, Nat. Mater. 12, 714 (2013).

[28] S. Tagliati, V. M. Krasnov, and A. Rydh, Differential membrane-based nanocalorimeter for high-resolution measurements of low-temperature specific heat, Rev. Sci. Instrum. 83, 055107 (2012).
[29] K. Nishimoto, T. Sato, and R. Tamura, Low-temperature superstructures of a series of $\mathrm{Cd}_{6} \mathrm{M}(\mathrm{M}=\mathrm{Ca}, \mathrm{Y}, \mathrm{Sr}, \mathrm{Pr}, \mathrm{Nd}, \mathrm{Sm}$, Gd, Tb, Dy, Ho, Er, Tm, Yb and Lu) crystalline approximants, J. Phys.: Condens. Matter 25, 235403 (2013).

[30] A. P. Ramirez, Strongly geometrically frustrated magnets, Annu. Rev. Mater. Sci. 24, 453 (1994).

[31] D. C. Joshi, G. Gebresenbut, C. Pay Gomez, and R. Mathieu, Memory and rejuvenation in a quasicrystal, Europhys. Lett. 132, 27002 (2020).

[32] T. Yamada, H. Takakura, T. Kong, P. Das, W. T. Jayasekara, A. Kreyssig, G. Beutier, P. C. Canfield, M. de Boissieu, and A. I. Goldman, Atomic structure of the $\mathrm{i}-R$-Cd quasicrystals and consequences for magnetism, Phys. Rev. B 94, 060103(R) (2016).

[33] T. J. Sato, S. Kashimoto, C. Masuda, T. Onimaru, I. Nakanowatari, K. Iida, R. Morinaga, and T. Ishimasa, Neutron scattering study on spin correlations and fluctuations in the transition-metal-based magnetic quasicrystal $\mathrm{Zn}-\mathrm{Fe}-\mathrm{Sc}$, Phys. Rev. B 77, 014437 (2008).

[34] Y. Tokiwa, C. Stingl, M.-S. Kim, T. Takabatake, and P. Gegenwart, Characteristic signatures of quantum criticality driven by geometrical frustration, Sci. Adv. 1, e1500001 (2015).

[35] H. Zhao, J. Zhang, M. Lyu, S. Bachus, Y. Tokiwa, P. Gegenwart, S. Zhang, J. Cheng, Y.-f. Yang, G. Chen, Y. Isikawa, Q. Si, F. Steglich, and P. Sun, Quantum-critical phase from frustrated magnetism in a strongly correlated metal, Nat. Phys. 15, 1261 (2019).

[36] D. Stauffer and A. Aharony, Introduction To Percolation Theory: Second Edition (2nded.) (Taylor \& Francis, London, 1992). 